\title{
Non-convex constrained economic power dispatch with prohibited operating zones and piecewise quadratic cost functions
}

\author{
Shaimaa R. Spea \\ Electrical Engineering Department, Faculty of Engineering, Menoufiya University, Egypt
}

\section{Article Info}

Article history:

Received Sep 25, 2019

Revised Mar 13, 2020

Accepted Mar 31, 2020

\section{Keywords:}

Crow search algorithm

Non-convex economic power

dispatch

Piecewise cost function

Prohibited operating zones

\begin{abstract}
This paper is focused on the solution of the non-convex economic power dispatch problem with piecewise quadratic cost functions and practical operation constraints of generation units. The constraints of the economic dispatch problem are power balance constraint, generation limits constraint, prohibited operating zones and transmission power losses. To solve this problem, a meta-heuristic optimization algorithm named crow search algorithm is proposed. A constraint handling technique is also implemented to satisfy the constraints effectively. For the verification of the effectiveness and the superiority of the proposed algorithm, it is tested on 6-unit, 10-unit and 15-unit test systems. The simulation results and statistical analysis show the efficiency of the proposed algorithm. Also, the results confirm the superiority and the high-quality solutions of the proposed algorithm when compared to the other reported algorithms.
\end{abstract}

Copyright $@ 2020$ Institute of Advanced Engineering and Science. All rights reserved.

Corresponding Author:

Shaimaa R. Spea,

Electrical Engineering Department, Faculty of Engineering,

Menoufiya University,

Shibin el Kom, Menofia Governorate, Egypt.

Email: shi_spea@yahoo.com

\section{INTRODUCTION}

Economic power dispatch (EPD) is an essential problem related to the electric power system. The objective of EPD is to obtain the optimal output power from each generation unit to minimize the fuel cost while satisfying all unit and system constraints [1]. In practical operating conditions of the power system, many thermal generation units are supplied with different fuels, multiple fuel options (MFO), like coal, oil, and natural gas. It is necessary to find the most economical fuel to be used in these units [2]. Also, the generation units may have prohibited operating zones (POZs) where the operation is unwanted and should be avoided [3]. These zones divide the decision space into disjoint subsets forming a non-convex solution space [4]. The practical EPD with MFO and POZs is a non-convex, non-continuous and non-differentiable optimization problem with many equality and inequality constraints, which makes it very difficult to obtain the optimal solution of this problem [5].

Due to the importance of the EPD problem, various optimization algorithms have been implemented to find its optimal solution. The conventional optimization techniques like quadratic programming [6], Linear Programming [7] and Gradient Method [8] have been successfully applied to solve the convex EPD problem. But these methods often fail to obtain the best solutions to the non-convex problems as they assumed that the functions are smooth and convex. Also, the convergence of these methods depends on the initial points, and they suffer from the probability of getting easily into the local search. Thus a lot of the conventional methods are not efficient in solving the EPD problem, especially when the practical conditions are considered [9]. Therefore, other effective search algorithms are required. 
Recently, the nature-inspired meta-heuristic search algorithms become popular in solving many real world optimization problems due to their promising performance, especially when the optimization problem is complex, non-linear, and multi-dimensional. Some of the meta-heuristic algorithms are cuckoo search [9, 10], elephant herding optimization [11], moth search algorithm [12], parallel hurricane optimization algorithm [13], particle swarm optimization [14], krill herd algorithm [15], earthworm optimization algorithm [16] and crow search algorithm (CSA) [17-19]. The CSA is simple in structure, easy to implement, and has fewer parameters that need settings. Also, the accuracy of results and time of convergence of CSA is better than the other algorithms like GA and PSO, which motivates it to find the solution of many optimization problems in the power system [17].

The purpose of the paper is to apply the CSA to find the solution to the non-convex constrained EPD problem. The multiple fuel options, prohibited operating zones, and transmission power losses are considered in the problem formulation of EPD. Also, a constraint handling technique is proposed to satisfy the different system constraints successfully. To show the efficiency of the proposed algorithm, it is applied to three vastly-used test systems with different economic load dispatch configurations, and the results are compared to other relevant reported algorithms. The other sections of the paper are arranged as follows: The mathematical model of the non-convex EPD problem is given in section 2. The description of the proposed CSA is presented in section 3. Section 4 explains the proposed constraint handling technique. The simulation results, statistical analysis and comparison results are discussed in section 5. Section 6 shows the conclusion of the paper.

\section{PROBLEM FORMULATION}

The EPD with MFO is an optimization problem that objects to minimize the total fuel cost among the multiple fuels of each unit while satisfying the different constraints. To model the MFO, the piecewise quadratic function is used to represent the fuel cost function. The EPD with MFO problem can be mathematically represented as follows:

$$
\begin{aligned}
& \min F_{\text {total }}=\sum_{i=1}^{N G} F_{i}\left(P_{G i}\right) \\
& F\left(P_{G i}\right)=\left\{\begin{array}{cc}
\alpha_{i 1}+\beta_{i 1} P_{G i}+\gamma_{i 1} P_{G i}^{2} & P_{G i}^{\min } \leq P_{G i} \leq P_{G i 1} \text { for fuel } 1 \\
\alpha_{i 2}+\beta_{i 2} P_{G i}+\gamma_{i 2} P_{G i}^{2} & P_{G i 1} \leq P_{G i} \leq P_{G i 2} \quad \text { for fuel } 2 \\
\cdot & \cdot \\
\alpha_{i L}+\beta_{i L} P_{G i}+\gamma_{i L} P_{G i}^{2} & P_{G i L-1} \leq P_{G i} \leq P_{G i}^{\max } \text { for fuel } L
\end{array}\right.
\end{aligned}
$$

where $\alpha_{i L}, \beta_{i L}$ and $\gamma_{i L}$ are the cost coefficients of the $i$-th generation unit for the fuel type $L$.

This problem will be solved subject to the following constraints:

1) Power balance constraint: The total generated power must satisfy the load demand $\left(P_{D}\right)$ and transmission losses $\left(P_{\text {loss }}\right)[1]$. Hence,

$$
\sum_{i=1}^{N G} P_{G i}=P_{D}+P_{l o s s}
$$

The power losses can be computed from the Kron's loss formula ( $B$-coefficient) as follows [20]:

$$
P_{\text {loss }}=\sum_{i=1}^{N G} \sum_{j=1}^{N G} P_{i} B_{i j} P_{j}+\sum_{i=1}^{N G} P_{i} B_{o i}+B_{o o}
$$

2) Generation limits constraint: The real output power from generator unit $i$ must be within limits as follows [1]:

$$
P_{G i}^{\min } \leq P_{G i} \leq P_{G i}^{\max }, \quad i=1, \ldots \ldots, N G
$$


3) Prohibited operating zones constraint: The thermal units can have POZs where the operation is undesirable due to instability issues or physical operational limitations on machine components [21]. A unit with POZs does not have a continuous fuel-cost curve as the operation should be avoided in these zones. This type of functions can be mathematically described as:

$$
\begin{aligned}
& P_{G i}^{\min } \leq P_{G i} \leq P_{G i, j}^{L} \\
& P_{G i, j}^{U} \leq P_{G i} \leq P_{G i, j+1}^{L} \quad j=1: P Z_{i} \\
& \cdots \ldots \ldots . . \\
& P_{G i, P Z_{i}}^{U} \leq P_{G i} \leq P_{G i}^{\max }
\end{aligned}
$$

where $P_{G i, j}^{L}$ and $P_{G i, j}^{U}$ are lower and upper bounds of the $j$-th POZ of unit $i$ and $P Z_{i}$ is the number of POZs of generation unit $i$.

\section{CROW SEARCH ALGORITHM}

Crow search algorithm is a meta-heuristic optimization algorithm presented by Askarzadeh in 2016 [17]. The main idea of the CSA is got from observing the social behavior of crows, which characterized by their intelligence. These birds live in the form of flocks. They have an excellent memory [18]. Crows observe the other birds, including the other crow members in the flock and watch where they hide their food. Then, they stole that food once the other birds left. The surplus food is hidden in a hideout spot and is restored when the crow needs it [19]. It is difficult to discover where the crow stored its food. If a crow observes another one goes after it, it will try to trick that crow and will go to another position [17]. This intelligent behavior of the crows is similar to the optimization process, and CSA attempts to simulate that behavior to find the optimal solutions to the optimization problems [17].

If we have a solution space with dimension $d$ that has a flock of $n$ crows, then the position $X$ of crow $i$ at iteration $t$ can be expressed by the vector:

$$
X^{i, t}=\left[x_{1}^{i, t}, x_{2}^{i, t}, \ldots \ldots, x_{d}^{i, t}\right]
$$

where $i=1: n, t=1: t^{\max }$ and $t^{\max }$ is the maximum number of iterations.

Each crow has a memory $m^{i, t}$ in which it stores the best position of its storing food source. The vector $X$ has the random initial positions of the crows. These positions are updated at each iteration, and this process is repeated until the stopping criterion is met. There are two cases for the positions update of the crows $[17,19]$ :

Case 1: Crow $j$ does not observe that crow $i$ is chasing it; hence, crow $i$ will get close to the hideout spot of crow $j$. In this state, the position of crow $i$ will be updated as follows:

$$
X^{i, t+1}=X^{i, t}+r a_{i} \times f l^{i, t} \times\left(m^{j, t}-X^{i, t}\right)
$$

where $r a_{i}$ is a random number with uniform distribution and its value between 0 and $1 . f l^{i, t}$ is the flight length of crow $i$ at iteration $t$.

Case 2: Crow $j$ observes that crow $i$ is chasing it. So, it will move to another position to trick crow $i$ and save its food. The two cases can be summarized as follows:

$$
X^{i, t+1}=\left\{\begin{array}{cc}
X^{i, t}+r a_{i} \times f l^{i, t} \times\left(m^{j, t}-X^{i, t}\right) & r_{j} \geq A P^{j, t} \\
\text { random position } & \text { otherwise }
\end{array}\right.
$$

where $r_{j}$ is the uniform distributed random number in the range of $[0,1]$ and $A P$ is the awareness factor. Figure 1 shows the main steps of the CSA for EPD problem. 


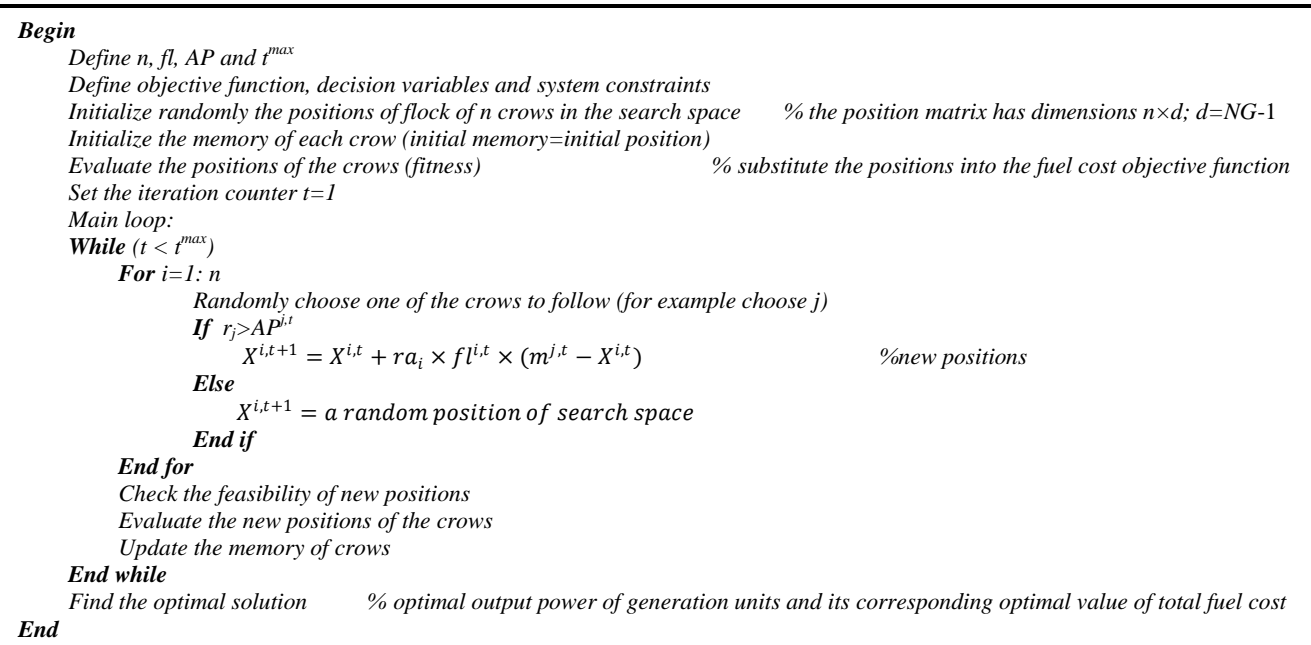

Figure 1. Steps of the proposed CSA for EPD problem

\section{CONSTRAINTS HANDLING TECHNIQUE}

In this section, the proposed constraints handling technique is explained with details. It is a necessary step to show how the CSA is applied to deal with the different constraints of the EPD problem.

a. Generators limit constraint: Since the control variables (power generation output) are created within their limits in the initialization step of the CSA, they indeed satisfy the corresponding inequality constraints.

b. Power balance constraint: To satisfy this constraint, one of the generators (control variables) is selected as a slack generator $P_{s}$ (dependent variable). Only $N G-1$ control variables are generated within their limits in the initialization step of the proposed CSA. $P_{s}$ value will be calculated as follows:

- In case of neglecting power losses: as follows:

The value of the slack generator, which satisfies this constraint, can be found by rewriting (3)

$$
P_{S}=P_{D}-\sum_{i=1}^{N G-1} P_{G i}
$$

- In case of considering power losses [22]:

The slack generator can be calculated as follows:

$$
P_{S}=P_{D}+P_{\text {loss }}-\sum_{i=1}^{N G-1} P_{G i}
$$

Substituting with (11) in (4), the power losses can be calculated as:

$$
P_{\text {loss }}=\sum_{i=1}^{N G-1} \sum_{j=1}^{N G-1} P_{i} B_{i j} P_{j}+2 P_{s}\left(\sum_{i=1}^{N G-1} B_{s i} P_{i}\right)+B_{s s} P_{s}^{2}+\sum_{i=1}^{N G-1} P_{i} B_{o i}+B_{o s} P_{s}+B_{o o}
$$

Expanding and rearranging, (11) becomes

$$
\begin{aligned}
& B_{s S} P_{s}^{2}+\left(2 \sum_{i=1}^{N G-1} B_{s i} P_{i}+B_{o s}-1\right) P_{S}+\left(P_{D}+\sum_{i=1}^{N G-1} \sum_{j=1}^{N G-1} P_{i} B_{i j} P_{j}+\sum_{i=1}^{N G-1} B_{o i} P_{i}-\right. \\
& \left.\sum_{i=1}^{N G-1} P_{i}+B_{o o}\right)=0
\end{aligned}
$$

Equation (13) can be simplified as:

$$
A P_{s}^{2}+B P_{s}+C=0
$$

where

$$
\begin{aligned}
& A=B_{S S} \\
& B=\left(2 \sum_{i=1}^{N G-1} B_{S i} P_{i}+B_{o S}-1\right)
\end{aligned}
$$




$$
C=\left(P_{D}+\sum_{i=1}^{N G-1} \sum_{j=1}^{N G-1} P_{i} B_{i j} B_{j}+\sum_{i=1}^{N G-1} B_{o i} P_{i}+B_{o o}\right)
$$

Solving (14) using the standard algebraic method, the value of $P_{s}$ can be found as:

$$
P_{S}=\frac{-B \pm \sqrt{B^{2}-4 A C}}{2 A}, \text { where } B^{2}-4 A C \geq 0
$$

The positive root of (15) is the value of the slack generator, which satisfies the power balance constraint when the power losses are considered. After calculating $P_{s}$, its value should be checked against its limits. If the limits are violated, the following procedure is used:

$$
P_{s}^{\text {lim }}= \begin{cases}P_{s}^{\max } & \text { if } P_{s}>P_{s}^{\text {max }} \\ P_{s}^{\text {min }} & \text { if } P_{s}<P_{s}^{\text {min }} \\ P_{S} & \text { if } P_{s}^{\text {min }} \leq P_{s} \leq P_{s}^{\text {max }}\end{cases}
$$

The new objective function to be minimized is:

$$
\min F_{\text {total }}=\sum_{i=1}^{N G} F_{i}\left(P_{G i}\right)+H_{p}\left(P_{s}-P_{s}^{l i m}\right)^{2}
$$

where $H_{p}$ is a penalty factor used to penalize the violated solutions.

c. Prohibited operating zones constraint: If POZs constraint is violated, then the generator output power $\left(P_{G i}\right)$ will be set to the nearest boundary of its POZs. This can be done by computing the mid-points of the POZs for each generator [23] as follows:

$$
\begin{aligned}
& M P_{i, k}=\frac{P_{G i, k}^{L}+P_{G i, k}^{U}}{2} k=1,2, \ldots ., P Z_{i} \\
& P_{G i}=P_{G i, k}^{L} \quad \text { if } P_{G i}<M P_{i, k} \\
& P_{G i}=P_{G i, k}^{U} \quad \text { if } P_{G i}>M P_{i, k}
\end{aligned}
$$

\section{SIMULATION RESULTS AND DISCUSSION}

To study the performance of the proposed CSA, it is applied to three vastly-used test systems with different EPD configurations. The proposed CSA is performed in MATLAB 7.10.0 environment. The programs are run on a personal computer with an Intel Core I5, $2.2 \mathrm{GHz}$ processor, $4 \mathrm{~GB}$ RAM and the Windows 8.1 operating system.

\subsection{Tuning parameters for proposed CSA}

Like other optimization algorithms, CSA requires tuning the parameters to ensure the efficient implementation of the algorithm. Unfortunately, this process is time-consuming, and if the number of parameters increases, the tuning time will increase. One advantage of the proposed CSA over many optimization algorithms that it has only two main parameters that need tuning, which are $A P$ and $f l$. The value of $A P$ controls the intensification and diversification of the optimization process. The opportunity of finding the storing food by the crows is increased when the value of $A P$ is decreased [17]. However, this opportunity is decreased when the value of $A P$ is increased as the crows search the space randomly [1]. The value of $f l$ affects the capability of the search [17]. The small values of $f l$ lead to local search, while the large values lead to a global search. A balance should be made between local and global searches. Setting the proper value of $f l$ helps in the convergence of the search algorithm [1] as the CSA may converge into local search, especially for multi-model problems. To optimize the parameters of the proposed CSA, several experiments are run by varying the values of CSA parameters as follows: $A P$ is changed from 0 to 1 with a step 0.05 , and $f l$ is changed from 0 to 5 with a step 0.1 .

The value of one parameter is changed in its range while the other parameter is fixed. For each combination of the parameters, the EPD problem is solved, and the best value of the objective function is calculated. The best values of the parameters which gave the minimum cost are chosen as the optimal settings of control parameters. It is found that the best value for $A P$ is 0.1 for all test cases. However, the best value for $f l$ is 3.0 for test case 2 and 2.0 for other cases. 


\subsection{Test case 1: 6-unit test system}

This system has 6 generation units with a total load demand of $1263 \mathrm{MW}$. The data of the 6-unit test system and the $B$-coefficients values are adopted from [21]. In this case, the system power losses are considered, and POZs constraint is included. Every generation unit has two POZs. Table 1 lists the simulation results for this case. The optimal value of fuel cost obtained by the proposed CSA and the corresponding value of power losses are $15442 \$ / \mathrm{hr}$ and $12.20 \mathrm{MW}$, respectively. The statistical results obtained by the proposed CSA method are compared to some of the reported EPD solution methods, as shown in Table 2. It is noticed that the proposed CSA has succeeded to find better values for minimum and mean fuel cost over the other algorithms. The convergence characteristics of the proposed CSA is shown in Figure 2. It is obvious that the proposed CSA has good convergence characteristics as it converges in relatively fewer iterations.

Table 1. Dispatch results for 6-unit test system with POZs and transmission losses

\begin{tabular}{ll}
\hline Output power & CSA \\
\hline$P_{1}$ & 446.9956 \\
$P_{2}$ & 172.9307 \\
$P_{3}$ & 261.9827 \\
$P_{4}$ & 143.1165 \\
$P_{5}$ & 163.4566 \\
$P_{6}$ & 86.7619 \\
Min. fuel cost & 15442.0 \\
Losses & 12.200 \\
\hline
\end{tabular}

Table 2. Comparison of the statistical results of different methods for 6-unit test system

\begin{tabular}{llll}
\hline Method & Min. Cost $(\$ / \mathrm{hr})$ & Mean Cost $(\$ / \mathrm{hr})$ & Max. Cost $(\$ / \mathrm{hr})$ \\
\hline Proposed CSA & 15442.00 & 15442.00 & 15466.00 \\
PSO [24] & 15450.00 & 15454.00 & 15492.00 \\
GA [24] & 15459.00 & 15469.00 & 15524.00 \\
CSA [25] & 15449.57 & 15449.76 & 15449.85 \\
MCSA-1 [25] & 15449.25 & 15449.34 & 15449.62 \\
MCSA-2 [25] & 15449.22 & 15449.32 & 15449.51 \\
MCSA* [25] & 15449.17 & 15449.24 & 15449.39 \\
MCSA* [26] & 15449.89 & 15449.89 & 15449.89 \\
\hline
\end{tabular}

*MCSA [25]: Modified crow search algorithm; MCSA [26]: Modified cuckoo search algorithm

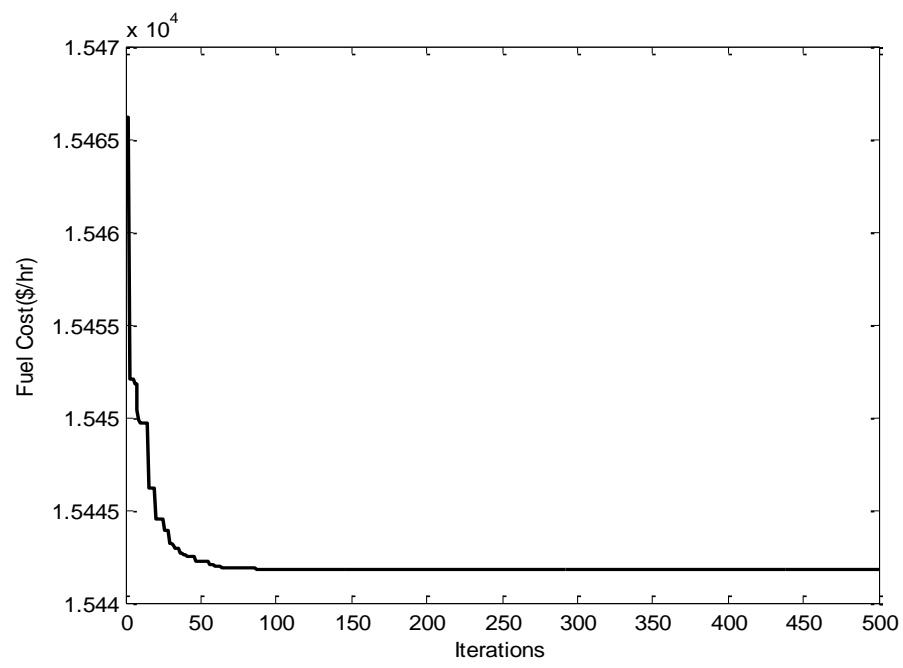

Figure 2. Convergence characteristics of the proposed CSA for test case 1

\subsection{Test case 2: 15-unit test system}

For further demonstration of the proposed CSA effectiveness, a larger practical system of 15-unit with POZs is used. The system power losses are neglected. The load demand is $2650 \mathrm{MW}$. The data of this test system is taken from [27]. The units 2, 5 and 6 have three POZs, and unit 12 has two POZs, which 
forming 192 decision sub-spaces. The simulation results are listed in Table 3. The optimal cost obtained is 32506.0 \$/hr. The comparison with other reported methods is listed in Table 4. Again, the proposed CSA proves its effectiveness in achieving the minimum value of fuel cost compared to the other reported methods.

Table 3. Simulation results for 15-unit test system with POZs

\begin{tabular}{llll}
\hline Unit & $P_{i}(\mathrm{MW})$ & Unit & $P_{i}(\mathrm{MW})$ \\
\hline$P_{1}$ & 454.9965 & $P_{9}$ & 25.0000 \\
$P_{2}$ & 455.0000 & $P_{I 0}$ & 20.0000 \\
$P_{3}$ & 130.0000 & $P_{11}$ & 60.0035 \\
$P_{4}$ & 130.0000 & $P_{12}$ & 75.0000 \\
$P_{5}$ & 260.0000 & $P_{13}$ & 25.0000 \\
$P_{6}$ & 460.0000 & $P_{14}$ & 15.0000 \\
$P_{7}$ & 465.0000 & $P_{15}$ & 15.0000 \\
$P_{8}$ & 60.0000 & - & - \\
Total Power Generated (MW) & & 2650.00 \\
Min. cost & & 32506.0 \\
\hline
\end{tabular}

Table 4. Comparison of the best fuel cost for 15 -unit test system

\begin{tabular}{ll}
\hline Method & Fuel Cost \\
\hline Proposed CSA & 32506.000 \\
IIA-MU [4] & 32544.982 \\
IA-MU [4] & 32544.991 \\
PM [27] & 32506.183 \\
DCGA [28] & 32516.169 \\
SGA [28] & 32513.219 \\
DP [28] & 32506.139 \\
GACOP [29] & 32544.970 \\
HPCSA [30] & $32,544.970$ \\
\hline
\end{tabular}

\subsection{Test case 3: 10-unit test system}

In this case, the efficiency of the proposed CSA method in solving non-smooth, non-convex EPD with POZs and MFO is proved. This test system has 10 generators. Each generator can be supplied by two or three different fuels. The system demand is varied gradually from $2400 \mathrm{MW}$ to $2700 \mathrm{MW}$. The transmission power losses are neglected for all load demands. The data of the system is taken from [3], and the data of POZs are given in Table 5. The dispatch results for the different loads and the fuel chosen for each generation unit are shown in Table 6. It is clear that the system constraints are handled successfully in all load demands, which ensures the efficiency of the proposed constraints handling technique. The results obtained by the proposed CSA are compared to DE [3], PSO [3] and QP-ALHN [3]. The comparison results are listed in Table 7. From this table, it is noticed that the proposed CSA can provide better results than DE and PSO in solving this problem.

Table 5. Prohibited operating zones for 10-unit test system

\begin{tabular}{|c|c|c|c|}
\hline Unit & Zone1 & Zone2 & Zone3 \\
\hline 3 & {$\left[\begin{array}{ll}215 & 225\end{array}\right]$} & {$\left[\begin{array}{lll}305 & 335\end{array}\right]$} & {$\left[\begin{array}{ll}420 & 450\end{array}\right]$} \\
\hline 5 & {$\left[\begin{array}{ll}200 & 220\end{array}\right]$} & {$\left[\begin{array}{ll}260 & 335\end{array}\right]$} & {$\left[\begin{array}{ll}390 & 420\end{array}\right]$} \\
\hline 7 & {$\left[\begin{array}{ll}230 & 255\end{array}\right]$} & {$\left[\begin{array}{ll}365 & 395\end{array}\right]$} & {$\left[\begin{array}{ll}430 & 455\end{array}\right]$} \\
\hline 10 & {$\left[\begin{array}{ll}270 & 295\end{array}\right]$} & {$\left[\begin{array}{ll}380 & 400\end{array}\right]$} & - \\
\hline
\end{tabular}

Table 6. Dispatch results for 10-unit test system with MFO and POZs at different load demands

\begin{tabular}{|c|c|c|c|c|c|c|c|c|}
\hline \multirow[b]{2}{*}{ Unit } & \multicolumn{2}{|c|}{$2400 \mathrm{MW}$} & \multicolumn{2}{|c|}{$2500 \mathrm{MW}$} & \multicolumn{2}{|c|}{$2600 \mathrm{MW}$} & \multicolumn{2}{|c|}{$2700 \mathrm{MW}$} \\
\hline & Fuel & $\mathrm{P}_{\mathrm{i}}(\mathrm{MW})$ & Fuel & $\mathrm{P}_{\mathrm{i}}(\mathrm{MW})$ & Fuel & $\mathrm{P}_{\mathrm{i}}(\mathrm{MW})$ & Fuel & $\mathrm{P}_{\mathrm{i}}(\mathrm{MW})$ \\
\hline 1 & 1 & 189.5595 & 2 & 207.2371 & 2 & 226.8055 & 2 & 215.6142 \\
\hline 2 & 1 & 202.2489 & 1 & 206.1971 & 1 & 213.4076 & 1 & 213.0907 \\
\hline 3 & 1 & 253.6248 & 1 & 265.6377 & 1 & 275.8827 & 1 & 278.2049 \\
\hline 4 & 3 & 232.9792 & 3 & 235.9278 & 3 & 247.2253 & 3 & 254.5116 \\
\hline 5 & 1 & 241.4600 & 1 & 258.0856 & 1 & 260.0000 & 1 & 260.0000 \\
\hline 6 & 3 & 232.9792 & 3 & 235.6990 & 3 & 239.0346 & 3 & 246.2529 \\
\hline 7 & 1 & 255.0000 & 1 & 268.9621 & 1 & 276.9065 & 1 & 283.9586 \\
\hline 8 & 3 & 232.9792 & 3 & 235.7410 & 3 & 246.7105 & 3 & 238.5308 \\
\hline 9 & 1 & 320.1298 & 1 & 331.3822 & 1 & 344.0272 & 3 & 439.8363 \\
\hline 10 & 1 & 239.0394 & 1 & 255.1303 & 1 & 270.0000 & 1 & 270.0000 \\
\hline TPG (MW) & \multicolumn{2}{|c|}{2400} & \multicolumn{2}{|c|}{2500} & \multicolumn{2}{|c|}{2600} & \multicolumn{2}{|c|}{2700} \\
\hline Min. cost & \multicolumn{2}{|c|}{481.7266} & \multicolumn{2}{|c|}{526.2388} & \multicolumn{2}{|c|}{574.7291} & \multicolumn{2}{|c|}{624.3212} \\
\hline
\end{tabular}


Table 7. Comparison of the best fuel cost for 10-unit test system with MFO and POZs

\begin{tabular}{ccccc}
\multicolumn{5}{c}{ at different load demands } \\
\hline Method & $2400 \mathrm{MW}$ & $2500 \mathrm{MW}$ & $2600 \mathrm{MW}$ & $2700 \mathrm{MW}$ \\
\hline Proposed CSA & 481.7266 & 526.2388 & 574.7291 & 624.3212 \\
DE [3] & 482.0683 & 526.4616 & 575.1903 & 624.6675 \\
PSO [3] & 482.0510 & 526.4546 & 574.9327 & 624.4452 \\
QP-ALHN [3] & 481.7266 & 526.2388 & 574.7291 & 624.3212 \\
\hline
\end{tabular}

\section{CONCLUSION}

In this paper, the CSA has been efficiently applied to solve the non-convex constrained EPD problem with multiple fuel options, prohibited operating zones, and transmission power losses. A constraint handling technique is successfully applied to handle different system constraints. Three vastly-used test systems; 6-unit, 10-unit, and 15-unit test systems have been considered. Three test cases are efficiently studied. The simulation results confirm the robustness and effectiveness of the proposed CSA. Also, the simulation results are compared to many reported algorithms. The comparison of results and the statistical analysis confirm the efficiency, high-quality solutions, and superiority of the proposed CSA to solve the practical constrained EPD problem.

\section{REFERENCES}

[1] R. Habachi, et al., "Solving economic dispatch and unit commitment problem in smart grid system using eagle strategy based crow search algorithm," Indonesian Journal of Electrical Engineering and Computer Science (IJEECS), vol. 14, no. 3, pp. 1087-1096, 2019.

[2] Y. V. K. Reddy and M. D. Reddy, "Grey Wolf Optimization for Solving Economic Dispatch with Multiple Fuels and Valve Point Loading, " International Journal of Information Engineering and Electronic Business, vol. 1, pp. 50-57, 2019.

[3] Vo N. Dieu and P. Schegner, "Augmented Lagrange Hopfield Network Initialized by Quadratic Programming for Economic Dispatch with Piecewise Quadratic Cost Functions and Prohibited Zones," Applied Soft Computing, vol. 13, pp. 292-301, 2013.

[4] C-L. Chiang, "Improved Immune Algorithm for Power Economic Dispatch Considering Units with Prohibited Operating Zones and Spinning Reserve," IACSIT International Journal of Engineering and Technology, vol. 6, no. 4, Aug. 2014.

[5] J. J. Q. Yu and V. O. K. Li, "A Social Spider Algorithm for Solving the Non-convex Economic Load Dispatch Problem," Neurocomputing, Jul. 2015.

[6] G. F. Reid and L. Hasdorff, "Economic dispatch using quadratic programming," IEEE Transactions on Power Apparatus and Systems, vol. PAS-92, no. 6, pp. 2015-2023, Nov. 1973.

[7] J. K. Delson and S. M. Shahidehpour, "Linear programming applications to power system economics, planning and operations," IEEE Transactions on Power Systems, vol. 7, no. 3, pp. 1155-1163, Aug. 1992.

[8] C. Yaşar and S. Fadil, "Solution to environmental/economic dispatch problem by using first order gradient method," in ELECO'2007 conference, Bursa, Turkey, Dec. 2007.

[9] R. Habachi, et al., "Economic and emission dispatch using cuckoo search algorithm," International Journal of Electrical and Computer Engineering (IJECE), vol. 9, no. 5, pp. 3384-3390, Oct. 2019.

[10] Z. Cui, et al., "A novel oriented cuckoo search algorithm to improve DV-Hop performance for cyber-physical systems," Journal Parallel Distributed Computing, vol. 103, pp. 42-52, 2017.

[11] G-G. Wang, et al., "A new metaheuristic optimization algorithm motivated by elephant herding behavior," International Journal Bio-Inspired Computation, vol. 8, no. 6, pp. 394-409, 2016.

[12] G-G. Wang, "Moth search algorithm: a bio-inspired metaheuristic algorithm for global optimization problems," Memetic Computing, vol. 10, no. 2, pp. 151-164, Jun. 2018.

[13] R. M. Rizk-Allah, et al., "A novel parallel hurricane optimization algorithm for secure emission/economic load dispatch solution', Applied Soft Computing, vol. 63, pp. 206-222, Feb. 2018.

[14] G-G. Wang, et al., "A novel improved accelerated particle swarm optimization algorithm for global numerical optimization," Engineering Computations, vol. 31, no. 7, pp. 1198-1220, 2014.

[15] L. Guo, et al., "A new improved krill herd algorithm for global numerical optimization," Neurocomputing, vol. 138, pp. 392-402, 2014.

[16] G-G. Wang, et al., "Earthworm optimization algorithm: a bio-inspired metaheuristic algorithm for global optimization problems," International Journal of Bio-Inspired Computation, vol. 12, no. 1, pp. 1-22, 2018.

[17] A. Askarzadeh, "A novel metaheuristic method for solving constrained engineering optimization problems: Crow search algorithm," Computers and Structures, vol. 169, pp. 1-12, 2016.

[18] R. Habachi, et al. "Resolution of economic dispatch problem of the moroccan network using crow search algorithm," Indonesian Journal of Electrical Engineering and Computer Science (IJEECS), vol. 13, no. 1, pp. 347-353, Jan. 2019,

[19] B. Z. Dr-Asli, et al., "Chapter 14: Crow Search Algorithm (CSA)," Advanced Optimization by Nature-Inspired Algorithms, Jul. 2017. 
[20] W. Khamsen, et al., "Hybrid method for solving the non smooth cost function economic dispatch problem," International Journal of Electrical and Computer Engineering (IJECE), vol. 10, no. 1, pp. 609-616, Feb. 2020.

[21] I. N. Trivedi and A. H. Ranpariya, "Economic Load Dispatch Problem with Ramp Rate Limits and Prohibited Operating Zones Solve using Levy Flight Moth-Flame Optimizer," in IEEE 2016 International Conference on Energy Efficient Technologies for Sustainability (ICEETS), 2016.

[22] A. Bhattacharya, "Hybrid Differential Evolution with Biogeography-Based Optimization for Solution of Economic Load Dispatch," IEEE Transactions on Power Systems, vol. 25, no. 4, Nov. 2010.

[23] P. K. Hota and N. C. Sahu, "Non-Convex Economic Dispatch with Prohibited Operating Zones through Gravitational Search Algorithm," International Journal of Electrical and Computer Engineering (IJECE), vol. 5, no. 6, pp. 1234-1244, Dec. 2015.

[24] A. A. El-Fergany, "Solution of Economic Load Dispatch Problem with Smooth and Non-Smooth Fuel Cost Functions Including Line Losses Using Genetic Algorithm," International Journal of Computer and Electrical Engineering (IJECE), vol. 3, no. 5, Oct. 2011.

[25] F. Mohammadi, H. Abdi, "A modified crow search algorithm (MCSA) for solving load dispatch problem," Applied Soft Computing, vol. 71, pp. 51-65, 2018.

[26] J. Zhao, et al., "Modified cuckoo search algorithm to solve economic power dispatch optimization problems," IEEE/CAA Journal of Automatica Sinica, vol. 5, no. 4, pp. 794-806, Jul. 2018.

[27] N. Çetinkaya, et al., "A New Algorithm and Computation Approach for Economic Dispatch with Prohibited Operating Zones in Power Systems," Energy Exploration \& Exploitation, vol. 23, no. 4, pp. 267-276, 2005.

[28] S. O. Orero and M. R. Irving, "Economic Dispatch of Generators with Prohibited Operation Zones: A Genetic Algorithm Approach," IEE Proc. Generation Transmission Distribution, vol. 143, no. 6, pp. 529-534, 1996.

[29] K. Min, et al., "An Economic Dispatch Algorithm as Combinatorial Optimization Problems," International Journal of Control, Automation, and Systems, vol. 6, no. 4, pp. 468-476, 2008.

[30] T. T. Nguyen, et al., "Modified Cuckoo Search Algorithm: A Novel Method to Minimize the Fuel Cost," Energies, vol. 11, no. 6, 2018. 